\title{
Cognitive and Neural Prerequisites for Time in Language: Any Answers?
}

\author{
Marianne Gullberg \\ Max Planck Institute for Psycholinguistics \\ Radboud University Nijmegen \\ Peter Indefrey \\ Max Planck Institute for Psycholinguistics \\ F.C. Donders Centre for Cognitive Neuroimaging
}

In the position article to this volume, Klein outlines a set of questions that are relevant for furthering the linguist's understanding of what the cognitive and neural prerequisites for time in language might be. He also declares a certain skepticism regarding the likelihood that new methods from other disciplines will provide answers to those questions. The articles in this issue, a selection of state-of-the-art findings concerning time from different disciplines and theoretical frameworks, all address some aspects of those questions. This overview attempts to assess to what extent the questions asked by Klein in his opening statement have been answered by contributions from other disciplines.

As a preamble, a general observation is that there is an inherent ambiguity in the notion of "time in language." The relationship between time and language is twofold. On the one hand, language production and comprehension occur over time and the processes involved have to be temporally orchestrated. The temporal planning skills required for this complex task may be absent at the beginning of language acquisition (cf. McCormack \& Hoerl, this issue) or may become impaired in later acquired language impairments (cf. Wearden, this issue; Kolk, this issue). On the other hand, language refers to time in a variety of ways, as evidenced in the catalogues of linguistic means for expressing temporality and aspect (cf. Klein, this issue; Evans, this issue). These two perspectives can interact, as seen in Kolk's example of how impaired memory

Correspondence concerning this article should be addressed to Marianne Gullberg, Max Planck Institute for Psycholinguistics, P.O. Box 310, 6500 AH Nijmegen, The Netherlands. Internet: Marianne.gullberg@mpi.nl 
duration interferes with the simultaneous activation of information (about time) at the conceptual level and syntactic processing, all of which results in a problem with tense marking. This final overview will mainly focus on time in language in the first sense; that is to say, on what is neurocognitively required in order for the linguistic means of expressing time to be deployed in actual language use.

\section{How Do Our Notions of Time and Our Ways to Express Them Change Over the Life Span?}

One reading of Klein's (this issue) first question concerning change in temporal representations is to query the relationship between cognitive maturity and language and, specifically, what the necessary and sufficient cognitive prerequisites for time in language may be. That is to say, what do we need to know or understand about time to be able to speak "correctly"? The article by McCormack and Hoerl (this issue) highlights the complex interplay between temporal cognition and linguistic skills. They focus on the development of socalled temporal decentering. This notion is defined not only by the coordination of temporal locations of three events (past, present, future) but also by what the authors call "event-independent thought about time," meaning the ability to think about events independently from familiar settings and concrete events in time. This research presupposes that coordination and event-independent thought are necessary prerequisites for appropriate (and adultlike) use of time in language. Based on what is known about the use of tense, the requirement concerning coordination of time points seems well founded. However, there may be reasons to doubt the necessity of event-independent thought, (i.e., the detachment of events from abstract positions on the time line). Consider, for instance, the use of past tense, which is primarily used to report on events from memory. Although adults may be capable of conceiving of event-free time positions, the storage of events in memory does not appear to make use of time tags referring to an event-independent time representation. Burt (this issue) reports on studies on autobiographical memory that show that the recall of past events does not rely on an abstract "calendric" system. Rather, recall relies on the temporal anchoring of a target event to other, highly salient events such as the starting points of "life themes" ("before I got married") or culturally shared events ("when Blair became prime minister"). Furthermore, autobiographical memory develops in parallel to linguistic development, such that both memory for and language about time seem to rely on more concrete instantiations of events and more on event-dependent temporal concepts than event-independent ones. In other words, the content of memory does not appear to have an 
event-independent temporal structure in storage or in retrieval. Eventindependent thought about time, supposedly the hallmark of adult, mature temporal cognition, may therefore not be necessary to talk appropriately about time.

Further to this, regardless of what the properties of adult temporal cognition may be, data from adult second language (L2) speakers interestingly indicate that temporal cognitive maturity may be necessary but not sufficient for the linguistic treatment of time, as highlighted by both Ellis (this issue) and Roberts (this issue). Adult L2 learners possess mature temporal cognition and a fully developed system for dealing with time in their first language (L1). Despite their cognitive and linguistic sophistication, they nevertheless display difficulties expressing time in the L2. Importantly, their difficulties are reminiscent of the difficulties connected to temporal decentering in children acquiring an L1 (e.g., expressing aspect before tense).

These observations together suggest a more complex relationship between the necessary and sufficient cognitive prerequisites for the treatment of time in language than is generally assumed, with the details of the relationship among (mature) temporal cognition, memory, and linguistic details still remaining to be defined.

\section{How Culture-Specific Are Our Notions of Time?}

Assuming a broad sense of "culture" and "notions of time," the answer to this question can only be "very culture-specific." Notions of time such as its granularity (days, hours, seconds or milliseconds), its cyclicity, or its importance for everyday life obviously differ widely across cultures and are also subject to historical changes. These changes are driven, for instance, by advances in the measurement of time, the availability of precise clock times to everyone, and by the role of time in industrial production processes.

Casasanto (this issue) provides a much less obvious answer to a much narrower question: How language-specific are our nonlinguistic representations or notions of temporal duration? More specifically, are they language-specific at all? The answer is "yes." Casasanto's experiments provide evidence that speakers of languages with a preference for duration-as-length metaphors (e.g., a long meeting), such as English, and speakers of languages with a preference for duration-as-quantity metaphors (e.g., much time), such as Greek, are differentially affected by length or quantity distracters in a nonverbal duration judgment task. Interestingly, Casasanto also shows that this effect is not culture-specific but only language-specific, in that English speakers 
behave like Greek speakers when trained to use more duration-as-quantity metaphors.

\section{How Are Events Located in Time in Other Domains of Cognition?}

Two relevant domains of cognition are memory, where past events are stored, and action planning, which deals with future events. Although some past events that are part of our semantic knowledge are stored with calendric tags (e.g., World War II 1939-1945), this is not how autobiographical events are stored in memory, as suggested by the studies summarized by Burt (this issue). When participants are asked to retrieve autobiographical events from memory, they typically fail to do so when prompted with a date cue ("What happened in your life on the 21st July 1976?"). Similarly, when asked to provide the date of a personal or public event, their results are poor. Events do not only seem to be stored without a calendric tag, but they also do not seem to be stored with information about their relative order. When asked about the order of personal events recorded in a diary about 4 months previously, a high proportion of participants' answers were erroneous (Burt, Kemp, Grady, \& Conway, 2000). Based on the general finding that an event's location in time is not stored in memory, Burt concludes that the location in time is reconstructed upon retrieval from memory. He suggests three mechanisms for this reconstruction. The first mechanism exploits the fact that although most autobiographical events do not have calendric tags, some important ones do (so-called "landmark" events, personal or public). Furthermore, events can be linked to "life themes" such as "the time I went to school," which often have at least approximate standard locations in time (and standard durations), which are part of our "autobiographical knowledge." Thus, linking some autobiographical event to a life theme and possibly even a landmark event (e.g., the day of graduation) allows for successful reconstruction of an event's approximate location in time.

The second mechanism makes use of the decay of memory traces (cf. Wearden, this issue; Tendolkar, this issue). Some estimate of the remoteness of an event can be obtained from the quality of the recollection. An interesting side observation in this context is that language generally is much less interested in the duration than in the order of events. Although languages can express (often calendric) duration estimation lexically (next week, last Friday, for 3 days), it is rare in the languages of the world for duration estimation to be part of the grammaticized, closed-class devices (cf. Klein, 1994). That said, some languages do make "remoteness" distinctions in (past) tense morphology, marking the temporal distance between time of speech and topic time. 
Often the distinction is between "today" and "before today." A few languages make more distinctions, such as Yagua, an Amazonian language that distinguishes five levels ranging from "a few hours before the time of the utterance," over "roughly a week to a month ago," to "distant or legendary past" (Dahl \& Velupillai, 2008; Payne \& Payne, 1990). To use languages with such remoteness distinctions in the past thus requires some duration or distance representation. However, it seems to require a level of detail that memory is not likely to provide. An interesting question is therefore how reconstruction might work in similar systems and whether the quality of memory traces plays a role here (Did something I did in the past happen 2 weeks ago or 8 weeks ago?). A further question is whether the use of a language marking duration requires the development of a different type of memory system. This proposal can be compared to the notion that the use of absolute spatial frames of reference, involving the use of cardinal directions and other geocentric units to locate entities in space (e.g., "spoon to north of cup"; Levinson, 2003, p. 48), rather than relative ones based on the speaker ("spoon to the right of cup") requires the ability to dead-reckon absolute location and orientation (Levinson). It would be interesting to conduct experiments similar to Burt's (this issue) in Yagua or other languages with remoteness distinctions to assess these issues.

A third mechanism suggested by Burt (this issue) allows for the reconstruction of smaller "episodic components" of an event. Although recall of the relative order of episodic components is generally poor, episodic components that are part of an event with a conventional temporal order of event components, such as a visit to a restaurant, can be stored and retrieved in the correct order due to our knowledge of that conventional order (although it may sometimes be difficult to decide whether a particular event component was recalled or confabulated according to the conventional event structure).

Turning to action planning and future events, Carota and Sirigu (this issue) discuss the relationship between future expressions in language and general notions of action planning at the neural level. They explore whether the processing of sequential goal-oriented actions in discourse and of sequential or future events as instantiated in word order draw on similar neural substrates or not (cf. Toni, this issue). Their findings suggest that although prefrontal cortex is implicated in the processing of sequential strings in both cases, the prefrontal cortex and Broca's area play differential roles in the processing of sequential strings for action and syntax, respectively. That is to say, the ordering of events and the processing of word orders expressing these events may not be directly linked at the neural level. 
Casasanto (this issue) discusses the relationship between another key conceptual category, namely space, and time, a relationship that has received a lot of attention (see references in Casasanto). The findings reviewed here suggest not only that the two categories display a close metaphorical relationship but also that (spatial) language reflects and also shapes temporal representations outside of linguistic practices. Overall, these findings suggest a permeable and fluid connection between general cognition and linguistic cognition concerning time.

\section{How Do the Various Devices, in Particular, Markings on the Verb and Temporal Adverbials, Interact?}

If this question is understood as referring to how temporal information encoded by tense and temporal adverbials is comprehended and integrated, then both Baggio's (this issue) semantic model and Zwaan's (this issue) simulation model have something to contribute. Baggio suggests that the interactions of the temporal meaning of lexical (adverbials) and grammatical devices (tense markers on the verb) are accounted for in a constraint satisfaction model. The system is incrementally confronted with different types of semantic constraints that need to be satisfied and unified for comprehension to take place. In (1), the constraints set up by the meaning of the adverbial (last Sunday), which is encountered first, must be unified with the meaning of the tense markings $(-s / e d)$ when these are encountered.

(1) Last Sunday Vincent *paints/painted the window frames of his country house.

If the temporal information specified in the adverbial (past) does not match the interval specified by the tense marking (present), inconsistency follows, unification fails, and the sentence is deemed meaningless. Interestingly, Klein (this issue) exemplifies the complex interaction between devices by a different type of construction, namely sentences containing combinations of tense specification and temporal adverbials (e.g., He has departed yesterday at four), which are deemed grammatically ill-formed in many languages on the grounds that a particular tense/aspect should not co-occur with temporal adverbials at all. Baggio's explicit semantic framework would find no fault with these types of sentences because there is no conflict between the temporal markers. The illformedness seems to reside outside of semantics. There is thus more linguistic work to do to account for these types of interactions. 
Zwaan (this issue) does not directly address the issue of the interaction of temporal adverbials and verb marking (cf. Evans, this issue). Nonetheless, he mentions two important roles for temporal adverbials for constructing event representations during language comprehension. In a mental model, event representations are indexed for the time at which the events occurred. Events that have the same time index (that happened at the same time) are more strongly connected in long-term memory. In stories and texts, temporal adverbials such as "a moment later" or "an hour later" provide information regarding whether two events share a time index. For example, Zwaan (1996) has shown that when participants are probed on a recognition task after reading a text, priming between events described as immediately successive (a moment later) is stronger than priming between events described as temporally separated (an hour later). Thus, in this role, temporal adverbials can interact with the memory encoding of events that are described by verbs.

In another role, temporal adverbials can interact more directly with aspectual markings on the verb, because both can modify the mental representation of an event. According to Zwaan (this issue), understanding the description of an event involving some action means performing a mental simulation of that action. Part of that simulation is a "motor resonance," essentially an activation of the listener's/reader's motor system that shares properties with the motor action in the described event. Zwaan suggests that aspectual distinctions can be reflected in such motor resonance. Thus, motor resonance may occur continuously throughout an imperfective sentence like He was painting the wall, but occur only on the verb in the corresponding perfective sentence He painted the wall. Importantly, motor resonance during language comprehension requires that all information that is relevant for the simulation be integrated until a "uniqueness point" is reached, where enough information for a simulation is available (cf. Evans, this issue). For example, in the sentence He slowly opened the door, motor activation corresponding to the opening of a door can only start when the door is reached (excluding other motor actions such as opening envelops). Note that in this example the modifier slowly is also part of the relevant information and will affect the exact form of the motor resonance. This means that in Zwaan's simulation model, all linguistic means that modify the meaning of an action operate in the same way, namely by modifying the corresponding motor resonance. This should also hold for temporal adverbials insofar as they affect the form of an action as in He held up his hand for a moment, then the waiter saw him compared to He held up his hand for ten minutes until the waiter saw him. 


\section{How Are Violations Within the Overall Expression of Time Handled?}

Baggio's article (this issue) is a most explicit answer to the question how violations within the expression of time are handled at the neurological level. Both his own electrophysiological (ERP) experiment and the experiment of Steinhauer and Ullman (2002), which he reviews, demonstrate that in response to sentences like (2) and (3), there are three distinct ERP responses: a left anterior negativity (LAN) starting as early as $200 \mathrm{~ms}$ after the violating verb, a late positivity (P600) starting 600-700 ms after the violating verb, and a sentence final negativity (SFN) following the last word of the sentence.

(2) Yesterday, I *sail Diane's boat to Boston. (Steinhauer \& Ullman, 2002)

(3) Afgelopen zondag *lakt Vincent de kozijnen van zijn landhuis. "Last Sunday Vincent *paints the window frames of his country house." (Baggio)

The complex electrophysiological response pattern alone suggests that "handling" violations is not one process but rather a set of processes happening at different time points following the violation. Baggio interprets the fastest response (LAN) as being driven by the detection of a feature mismatch (resulting in a failure of syntactic unification), analogous to known LAN/P600 response patterns to case, number or gender mismatches. This interpretation requires him to develop a theory of what the values of a tense feature may be. Note that the violating verbs were marked for tense so that the violation cannot simply consist in the absence of a tense marking but must be due to the temporal meaning of the tense marker and, hence, arise at a semantic level. Given that the violation response started already around $200 \mathrm{~ms}$ after the presentation of the verb, Baggio's conclusion that tense feature checking must be informed by semantic representations already at a syntactic unification stage seems valid. Nonetheless, Baggio's definition of a tense violation as a situation in which "the anomalous verb located the event described by the main clause outside a frame specified by an anchoring temporal expression" may be too constraining. Neither his findings nor the findings of other studies so far have investigated the effect of the absence of a tense marking where one is required (a phenomenon that is not uncommon but may even be typical of some learner varieties (cf. Klein \& Perdue, 1997). In addition to the observed "semantic" tense violation, there could be a purely "syntactic" tense violation in sentences like (4):

(4) Yesterday, I *sailing Diane's boat to Boston. 
Here, a nonfinite verb form appears instead of a finite, tense-marked verb form. Obviously, in English a nonfinite verb form lacks not only tense but also person and number features. To avoid this confound, such an experiment would have to be conducted in a language in which verb forms carry only tense but not person or number features (e.g., Japanese).

According to Baggio (this issue), the SFN reflects another processing stage where the temporal constraints set up by the verb are "readjusted" such that, for example, a "narrative present" reading of the sentence may result. This option points to a flexibility in Baggio's semantically informed tense features reminiscent of cases where syntactic violations of other semantically interpretable features are accepted or even preferred - for example, natural gender congruency in German (5) or number congruency as in English (6):

(5) Das Mädchen war stolz auf ihr neues Kleid.

"The girl $l_{\text {neuter }}$ was proud of her feminine $_{\text {new dress." }}$

(6) The police have arrested...

It is conceivable that the readjustment process suggested by Baggio (this issue) might also play a role for other features, such that a more general treatment of semantically interpretable syntactic features might result.

In contrast to a feature mismatch, this type of readjustment is not possible in the case of absence of a necessary tense feature in sentences like (4). This type of "tense" violation clearly has a different quality and might serve as a test case for the interpretation of the SFN. As no readjustment is possible, there should be no SFN if the readjustment is indeed reflected in this ERP effect.

In sum, Baggio's findings give a clear indication of how tense violations may be processed. Furthermore, his interpretation in terms of a theory of semantically informed tense feature checking provides a highly interesting account of tense (and perhaps not only tense) processing in general.

\section{Concluding Remarks}

This review on the whole suggests that evidence from other disciplines and theoretical frameworks do provide answers to some of the questions that Klein considers to be vital to the linguist whose daily bread consists of thinking about time and language. Other questions have answers under particular readings but leave other aspects of the questions open. Finally, in those cases in which no clear answers are to be had, the cross-disciplinary perspective and the different theoretical frameworks nevertheless seems to inspire new, interesting, and sometimes more specific questions than previously asked. This in turn suggests 
that, Klein's skepticism notwithstanding, findings and methods from other disciplines and domains can open fruitful new avenues of research to explore old issues.

\section{References}

Burt, C. D. B., Kemp, S., Grady, J. M., \& Conway, M. A. (2000). Ordering autobiographical experiences. Memory, 8, 323-332.

Dahl, Ö., \& Velupillai, V. (2008). The past tense. In M. Haspelmath, M. S. Dryer, D. Gil, \& B. Comrie (Eds.), The world atlas of language structures online (chapter 66). Munich: Max Planck Digital Library. Retrieved June 6, 2006, from http://wals.info/feature/66.

Klein, W. (1994). Time in language. London: Routledge.

Klein, W., \& Perdue, C. (1997). The basic variety (or: Couldn't natural languages be much simpler?). Second Language Research, 13, 301-347.

Levinson, S. C. (2003). Space in language and cognition: Explorations in cognitive diversity. Cambridge: Cambridge University Press.

Payne, D. L., \& Payne, T. (1990). Yagua. In D. C. Derbyshire \& G. K. Pullum (Eds.), Handbook of Amazonian languages (Vol. 2, pp. 249-474). Berlin: Mouton.

Steinhauer, K., \& Ullman, M. T. (2002). Consecutive ERP effects of morpho-phonology and morpho-syntax. Brain and Language, 83, 62-65.

Zwaan, R. A. (1996). Processing narrative time shifts. Journal of Experimental Psychology: Learning, Memory, and Cognition, 22, 1196-1207. 